\title{
Homotopy Sumudu Transformation Method for Solving Fractional Delay Differential Equations
}

\author{
N. R. Anakira ${ }^{a}, *$ H. Abdelkarimª, M. Abu-Dawas ${ }^{b}$ \\ ${ }^{a}$ Department of Mathematics, Faculty of Science \& Information Technology, Irbid National University, Jordan \\ ${ }^{b}$ Computer Science Department, Faculty of Science Information Technology, Irbid National University, Jordan
}

\begin{abstract}
In this article, A new accurate approximate solution for a nonlinear fractional delay differential equations are obtained using an effective algorithm so called homotopy analysis sumudu transformation method (HASTM). Some examples have been solved to demonstrate the methodology of this procedure. The accuracy of the obtained numerical results has been achieved via rapid rate of convergence for the obtained approximate solutions. Numerical comparison which was displayed and presented in tables and also shown graphically in figures prove and confirm the high accuracy, capability and efficiency of this procedure. (C2020 All rights reserved.
\end{abstract}

Keywords: Fractional Calclus, Sumudu Transform, Homotopy Analysis Method.

2010 MSC: 34A08, 58B05, 37Mxx .

\section{Introduction}

In the last years, there has been much great attention in the field of study fractional differential equations, since the modern research in applied science and engineering have demonstrate that the dynamic of many system are modeled using differential equations of non-integer order due to their broad applications in complex physical system and viscoelastic materials, fluid mechanics and electromagnetism $[26,13,25,4,14,1,5]$. Delay differential equations (DDEs) are considered as a kind of functional differential equations that has many applications in several areas of studies such as biology, economy, electrodynamics, control and quantum mechanics. DDEs are more general than classical differential equations because the derivative of the unknown function at certain time depend on the value of the function at previous time or equations which have delayed argument. In many field of applied science and engineering DDEs play an important role and numerous approximated and numerical methods have been employed to solve different classes of DDEs [28, 6, 11, 19, 24, 8, 9]. Recently, fractional delay differential equations (FDDEs) have attracted the attentions of researchers from all over the world in a wide range area of science and engineering such as ecology, physical science and groundwater flow. For instance, the following equation indicate to the food-limited model that relates the nourishment rage of area the effect of food on the population of that area in surveyed.

\footnotetext{
${ }^{*}$ Corresponding author

Email addresses: alanaghreh_nedal@yahoo.com ( N. R. Anakira), ( H. Abdelkarim), motasem_dawas@yahoo.com ( M. Abu-Dawas)

doi:10.31559/glm2020.9.1.4
} 


$$
{ }_{0} D_{x}^{\alpha} u(x)=r u(x)\left(\frac{k-u(x-\tau)}{k+m u(x-\tau)}\right), \quad u(x)=0.5(-\tau \leqslant x \leqslant 0)
$$

Also, the number of blood cells in human body can be represented by the following FDDE

$$
{ }_{0} D_{x}^{\alpha} u(x)=\frac{c a^{r} y(x-\tau)}{a^{r}+y^{r}(x-\tau)}-g y(x), \quad u(x)=0.02(-\tau \leqslant x \leqslant 0)
$$

Many authors studied FDDEs to obtain approximate solutions using various approximated analytical method such as HAM, OHAM, ADM and so on besides other type of differential equations of integer or non integer order $[30,18,15,27,29,23,20,31,7,16,21,2,22,3,10]$, but the most important challenge that faces the researchers and should be taken under consideration is to find efficient and more accurate procedure which gives exact or accurate results with a very low remarkable error. In this paper, an effective procedure which gives solutions in form of convergent series solutions with easily computations and without linearization or restrictive assumptions is presented.

This paper is summarized as follows, Section two, include the fundamental properties of fractional calculus and sumudu transform method. Section 3 is devoted for description of the basic idea of the HASTM. Section 4 is devoted to prove the validity and capability of this procedure by presenting and discussing some numerical experiments, Finally, we end by including the conclusion of this study in last Section.

\section{Fundamental Properties oF Fractional Calculus and Sumudu Transform Method}

This Section deals with some notation and basic definitions of the fractional calculus theory and Sumudu transform that will be used further in this paper([12]).

Definition 2.1. The real function $f(x), x>0$ said to be in the space $C_{\mu}, \mu \in \mathfrak{R}$ if there exists a real number $(p>\mu)$, such that $f(x)=x^{p} f_{1}(x)$, where $f_{1}(x) \in c[0, \infty)$ and it is said to be in the space $C_{\mu}^{m}$ iff $\left.f^{(} m\right) \in C_{\mu}$ where $m \in N$

Definition 2.2. The left sided Riemann-Liouville fractional integral operator of order $\alpha \geqslant 0$ of a function $f \in C_{\mu}, \mu \geqslant-1$ is defined as:

$$
\mathrm{J}_{\mathrm{a}}^{\alpha} \mathrm{f}(\mathrm{x})=\frac{1}{\Gamma(\alpha)} \int_{\mathrm{a}}^{x}(x-\tau)^{\alpha-1} f(\tau) \mathrm{d} \tau, x>0
$$

and $\Gamma(\alpha)$ is the gamma function.

Some properties of the operator $J^{\alpha}$, where $f \in C_{\mu}, \mu \geqslant-1, \alpha, \beta \geqslant 0$ and $\gamma>-1$

- $J_{a}^{\alpha} J_{a}^{\beta} f(x)=J_{a}^{\alpha+\beta} f(x)=J_{a}^{\beta} J_{a}^{\alpha} f(x)$

- $\mathrm{J}_{\mathrm{a}}^{\alpha} \mathrm{C}=\frac{\mathrm{c}}{\Gamma(\alpha+1)} \mathrm{t}^{\alpha}$, where $\mathrm{C}$ is constant

- $\mathrm{J}_{\mathrm{a}}^{\alpha} \mathrm{t}^{\gamma}=\frac{\gamma+1}{\Gamma(\alpha+\gamma+1)} \chi^{\alpha+\gamma}$

Definition 2.3. The caputo definition of fractional derivative operator is given by $D_{*}^{\alpha} a f(x)=J^{n-\alpha_{a} D^{n} f(x)}=$ $\left.\frac{1}{\Gamma(n-\alpha)} \int_{a}^{x}(x-\tau)^{n-\alpha-1} f^{(} n\right)(\tau) d \tau, t>0, \forall n-1<\alpha \leqslant n, n \in N$

Definition 2.4. The Sumudu transformation is defined as follows, Consider $\mid$ vjhgk $\mid$

Properties of the Sumudu transform are given as: 
- $\mathrm{S}[1]=1$

- $S\left[e^{a t}\right]=\frac{1}{1-a u}$

- $S\left[\frac{t^{n}}{\Gamma(n+1)}\right]=u^{n}, \quad n>0$

- $S[\alpha f(x)+\beta g(x)]=\alpha S[f(x)+\beta S[g(x)]$

Definition 2.5. If $G^{n}(u)$ is Summudu transformation of $n^{\prime}$ th order derivative of $f 6 n(t), \forall n \geqslant 1$ then we have:

$\mathrm{G}^{\mathrm{n}}(\mathrm{u})=\frac{\mathrm{F}(\mathrm{u})}{\mathrm{u}^{\mathrm{n}}}-\sum_{k=0}^{\mathrm{n}-1} \frac{\mathrm{f}^{\mathrm{k}}(0)}{\mathrm{u}^{\mathrm{n}-\mathrm{k}}}$

where $-1 \leqslant n-1 \leqslant \alpha<n$

Definition 2.6. The Sumudu transform $S[f(x)]$ of the fractional derivative introduced by Caputo is given by $S\left[D^{\alpha} f(x)\right]=\frac{S[f(x)]}{u^{\alpha}}-\sum_{k=0}^{n-1} \frac{f^{k}(0)}{u^{n-k}}, \quad-1<\alpha \leqslant n$

\section{Homotopy Analysis Sumudu transform Method}

To explain the basic idea of the homotopy analysis Sumudu transform method (HASTM) for the fractional delay differential equations [? ], we consider

$$
\mathrm{D}^{\alpha}\left[\mathrm{u}^{\prime}(x)\right]=\mathrm{N}[\mathrm{u}(\mathrm{x})]
$$

Applying Sumudu transform on both sides of equation (3.1), we have:

$$
\frac{S[u(x)]}{v^{\alpha}}-\sum_{n=0}^{m-1} v^{-\alpha+k} u^{k}(0)=S[N[u(x)]]
$$

and this yields

$$
\begin{aligned}
& S[u(x)]=\sum_{n=0}^{m-1} v^{+k} u^{k}(0)+v^{\alpha}[N[u(x)]] \\
& S[u(x)]=g\left(v, f_{i}\right)+v^{\alpha}[N[u(x)]]
\end{aligned}
$$

where $g\left(v, f_{i}\right)=f_{0}(x)+v f_{1}(x)+v^{2} f_{2}(x) v+\ldots+v^{m-1} f_{m-1}(x)$. Some time it's difficult to find $u(x ; t)$ by taking Sumudu inverse for both side of Eq. (3.4) particularly for nonlinear $N[u(x)]$. So we can utilized the HAM by defining the map:

$$
\left.(1-q) S[\phi(x ; q)]-u_{0}(x)\right]=h q H(x) N[u(x)]
$$

where $\mathrm{q} \in[0,1]$ is the embedding parameter and $\mathrm{H}(\mathrm{x} ; \mathrm{q})$ is real function of $\mathrm{x}$ and $\mathrm{q}, \mathrm{N}$ nonlinear operator defined by:

$$
\mathrm{N}[\phi(x ; q)]=S[\phi, q ;]-v^{\alpha}[\mathrm{N}[\mathrm{u}(\mathrm{x})]]-\mathrm{g}\left[v, \mathrm{f}_{\mathrm{i}}\right]
$$

Obeviosly, when $q \in[0,1]$ it's hold $\phi(x ; q)=u_{0}$ and also atq $=1$ it's hold $\phi(x ; q)=u(x)$. Thus as $q$ increase from 0 to 1 the solution various from the initial guess to the exact solution. We can expand $\phi(x ; q)$ in Taylor series with respect to $\mathrm{q}$ as

$$
\phi(x ; q)=u_{0}(x)+\sum_{m=1}^{\infty} u_{m}(x) q^{m}
$$


where $u_{m}(x)=\frac{1}{m !} \frac{\partial^{m} \phi}{\partial q^{m}}$

the convergent of the series solution depends on $h$, if we probably chose $h, u_{0}(x)$ and $H(x ; q)$, the series (3.7) convergent at $q=1$, hence, we obtain

$$
u(x)=u_{0}(x)+\sum_{m=1}^{\infty} u_{m}(x)
$$

which is a solution of original nonlinear equation, this equation represents the relationship between the initial guess $u_{0}(x)$ and the exact solution $u(x)$ by means of the term $u_{m}(x)$, which are still to be calculated. Defined the vector $\vec{u}=\left(u_{0}, u_{1}, \ldots, u_{m}\right)$. Differentiating the zeroth-order deformation equation (5.23) $\mathrm{m}$ times with respect to $q$ and then dividing them by $m$ ! and finally setting $q=0$, we get the following $m^{\prime}$ th-order deformation equation:

$$
S\left[u_{m}(x)-\chi_{m} u_{m-1}(x)\right]=h H(x) R_{m}\left(u_{m-1}\right)
$$

Applying the inverse sumudu transform, we have

$$
\left.u_{m}(x)=\chi_{m} u_{m-1}(x)\right]+h S^{-1}\left[H(x) R_{m}\left(\vec{u}_{m-1}\right)\right]
$$

where

$$
R_{m}(x) \vec{u}_{m-1}=\frac{1}{(m-1) !} \frac{\partial^{m-1} N[\phi(x ; q)]}{\partial q^{m-1}} \mid q=0
$$

and

$$
x_{m}= \begin{cases}0 & m \leqslant 1 \\ 1, & m>1\end{cases}
$$

\section{NUMERICAL RESULTS}

In this section, we investigate the effectiveness of the proposed procedure by solving several examples of fractional delay differential equations.

\subsection{Example 1}

Consider the following fractional delay differential equation [12]

$$
\mathrm{D}^{\alpha} \mathrm{u}^{\prime}(\mathrm{x})=\frac{3}{4} \mathrm{u}(\mathrm{x})+\mathrm{u}\left(\frac{x}{2}\right)-x^{2}+2, \quad 0 \leqslant x \leqslant 1, \quad 1<\alpha \leqslant 2,
$$

subjects to the initial conditions $u(0)=0, \quad u^{\prime}(0)=0$.

The exact solution for this problem in case of $\alpha=2$ is

$$
u(x)=x^{2} .
$$

Table 1 present a comparison between the absolute error obtained by HASTM approximate solution, Sumudu transform method and the exact solutions given by Eq. (4.2). It is easily observed from the results displayed in Table 1 that the approximate solution obtained by HASTM is more accurate than the approximate solution obtained by Sumudu transform method. Fig. 1 displays the plot of the exact solution and the approximate solutions for some values of $\alpha$, and also represents the plot of the $h-$ curve and the absolute error. 
Table 1: The approximate solution by HASTM at different values of $\alpha$, and comparison with exact solution and Sumudu transformation method for Example 1.

\begin{tabular}{llllll}
\hline$x$ & $\begin{array}{c}\text { Exact } \\
\text { Solution }\end{array}$ & $\begin{array}{c}\text { HASTM Solution } \\
\text { when } \alpha=1.5\end{array}$ & $\begin{array}{c}\text { HASTM Solution } \\
\text { when } \alpha=2\end{array}$ & $\begin{array}{l}\text { HASTM Absolute } \\
\text { error when } \alpha=2\end{array}$ & \multicolumn{2}{c}{$\begin{array}{c}\text { Absolute error } \\
\text { by when } \alpha=2 ~[12]\end{array}$} \\
\hline 0.1 & 0.01 & 0.0478911 & 0.01 & 0.000 & 0.00 \\
0.2 & 0.04 & 0.136917 & 0.04 & 0.0000 & 0.000 \\
0.3 & 0.09 & 0.254736 & 0.09 & 0.000 & 0.00 \\
0.4 & 0.16 & 0.397657 & 0.16 & 0.000 & 0.00 \\
0.5 & 0.25 & 0.563926 & 0.25 & 0.000 & 0.000001 \\
0.6 & 0.36 & 0.752611 & 0.36 & 0.0 .000 & 0.000006 \\
0.7 & 0.49 & 0.963211 & 0.48999 & $5.6 \times 10^{-17}$ & 0.000022 \\
0.8 & 0.64 & 1.19548 & 0.63999 & $5.6 \times 10^{-16}$ & 0.000064 \\
0.9 & 0.81 & 1.44934 & 0.08099 & $3.6 \times 10^{-15}$ & 0.000165 \\
1.0 & 1.0 & 1.72483 & 0.99999 & $1.9 \times 10^{-14}$ & 0.000383 \\
\hline
\end{tabular}

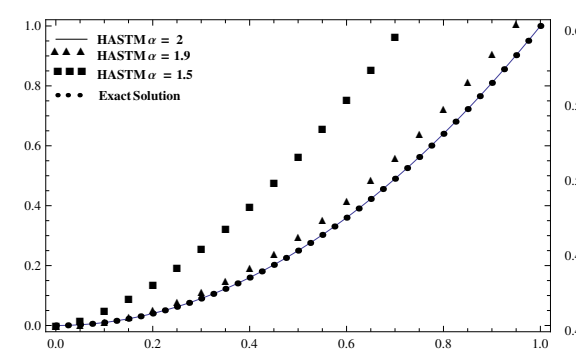

(a)

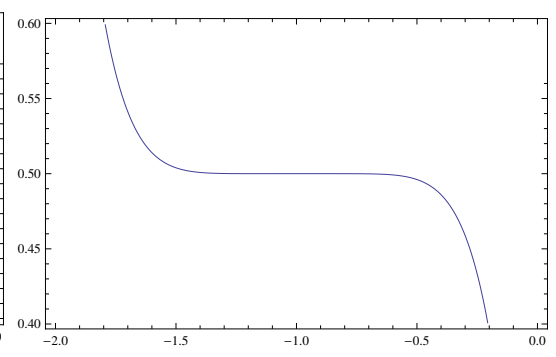

(b)

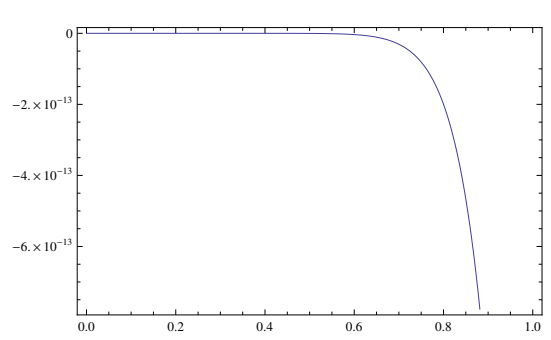

(c)

Figure 1: (a) Comparison between the approximate solution obtained by HASTM at different values of $\alpha$ and the exact one (b)Reprsents the $h$ curve (c) HASTM absolute error 
Table 2: The approximate solution by HASTM at different values of $\alpha$, and comparison with exact solution and Sumudu transformation method for Example 2.

\begin{tabular}{lllcll}
\hline$x$ & $\begin{array}{c}\text { Exact } \\
\text { Solution }\end{array}$ & $\begin{array}{c}\text { HASTM } \\
\alpha=0.75\end{array}$ & $\begin{array}{c}\text { HASTM } \\
\alpha=1\end{array}$ & $\begin{array}{c}\text { HASTM Absolute } \\
\alpha=1\end{array}$ & $\begin{array}{c}\text { Absolute error } \\
\alpha=1[12]\end{array}$ \\
\hline 0.1 & 0.01 & 0.01 & 0.01 & 0.00 & 0.000000 \\
0.2 & 0.04 & 0.04 & 0.04 & $5.9 \times 10^{-16}$ & 0.000000 \\
0.3 & 0.09 & 0.09 & 0.09 & $5.13 \times 10^{-14}$ & 0.000001 \\
0.4 & 0.16 & 0.16 & 0.16 & $1.21 \times 10^{-12}$ & 0.000007 \\
0.5 & 0.25 & 0.25 & 0.25 & $1.41 \times 10^{-11}$ & 0.000027 \\
0.6 & 0.36 & 0.36 & 0.36 & $1.05 \times 10^{-10}$ & 0.00008 \\
0.7 & 0.49 & 0.49 & 0.49 & $5.73 \times 10^{-10}$ & 0.0002 \\
0.8 & 0.64 & 0.64 & 0.64 & $2.49 \times 10^{-9}$ & 0.000444 \\
0.9 & 0.81 & 0.81 & 0.81 & $9.09 \times 10^{-9}$ & 0.000898 \\
1.0 & 1.0 & 1.0 & 1.0 & $2.9 \times 10^{-8}$ & 0.001686 \\
\hline
\end{tabular}

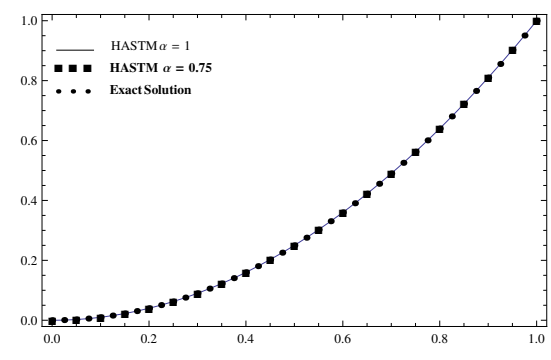

(a)

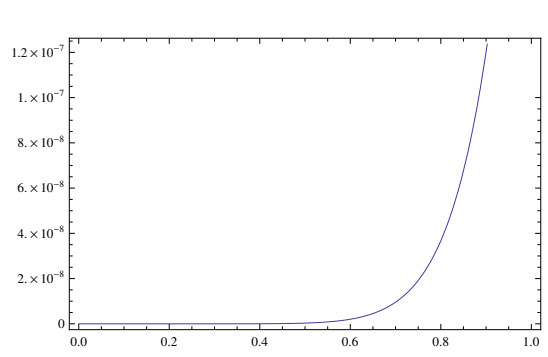

(b)

Figure 2: (a) Comparison between the approximate solution obtained by HASTM at different values of $\alpha$ and the exact one (b) HASTM absolute error

\subsection{Example 2}

Consider the following fractional delay equation [12]

$$
\mathrm{D}^{\alpha} \mathrm{u}^{\prime}(x)=-\mathrm{u}(\mathrm{x})+\mathrm{u}\left(\frac{x}{2}\right)+\frac{3}{4} x^{2}+\frac{2}{\Gamma(3-\alpha)} x^{2-\alpha}, \quad 0 \leqslant x \leqslant 1, \quad 0<\alpha \leqslant 1,
$$

subjects to the initial conditions $u(0)=0$, and exact solution $u(x)=x^{2}$ in case of $\alpha=1$

Table 2 shows the comparison of the absolute error between approximate solution obtained by HASTM and Sumudu transformation method while Figs. 2 (a) and (b) are displayed for the plot of the approximate solution, exact solution and the absolute error, respectively. The accuracy of the presented procedure is very clear compared the other method in literature, this leads to conclude and prove that the presented procedure is very powerful and suitable for this kind of differential equations.

\subsection{Example 3}

Consider the following fractional delay equation [17]

$$
\mathrm{D}^{\alpha} \mathrm{u}(\mathrm{x})=1-2 \mathrm{u}^{2}\left(\frac{x}{2}\right), \quad 0 \leqslant x \leqslant 1, \quad 0<\alpha \leqslant 2,
$$

subjects to the initial conditions $u(0)=1, \mathfrak{u}^{\prime}(0)=0$, and exact solution $u(x)=\cos x$ in case of $\alpha=1$

Table 3 present a comparison between the absolute error obtained by HASTM approximate solution, modified laguerre wavelets method and the exact solutions given by Eq. (4.2). It is easily observed from the results displayed in this Table that the approximate solution obtained by HASTM is more accurate 
Table 3: The approximate solution by HASTM at different values of $\alpha$, and comparison with exact solution and modified Laguerre wavelets method for Example 3.

\begin{tabular}{cccclc}
\hline$x$ & $\begin{array}{c}\text { Exact } \\
\text { Solution }\end{array}$ & $\begin{array}{c}\text { HASTM } \\
\alpha=0.75\end{array}$ & $\begin{array}{c}\text { HASTM } \\
\alpha=2\end{array}$ & $\begin{array}{c}\text { HASTM Absolute } \\
\alpha=1\end{array}$ & $\begin{array}{c}\text { Absolute error } \\
\alpha=1[17]\end{array}$ \\
\hline 0.1 & 0.995004165 & 0.988975813 & 0.995004165 & 0.00000 & $2.11000 \times 10^{-8}$ \\
0.2 & 0.980066578 & 0.963174957 & 0.980066578 & 0.000 & $2.09000 \times 10^{-8}$ \\
0.3 & 0.955336489 & 0.925890213 & 0.955336489 & 0.000 & $2.09000 \times 10^{-8}$ \\
0.4 & 0.921060994 & 0.878995420 & 0.921060994 & $1.11022 \times 10^{-16}$ & $2.08000 \times 10^{-8}$ \\
0.5 & 0.877582562 & 0.824018155 & 0.877582562 & $1.11022 \times 10^{-16}$ & $2.06000 \times 10^{-8}$ \\
0.6 & 0.825335615 & 0.762342705 & 0.825335615 & 0.000 & $2.04000 \times 10^{-8}$ \\
0.7 & 0.764842187 & 0.695278761 & 0.764842187 & $2.22045 \times 10^{-16}$ & $2.03000 \times 10^{-8}$ \\
0.8 & 0.696706709 & 0.624087729 & 0.696706709 & $1.33227 \times 10^{-15}$ & $2.00000 \times 10^{-8}$ \\
0.9 & 0.621609968 & 0.549991883 & 0.621609968 & $8.77076 \times 10^{-15}$ & $1.99000 \times 10^{-8}$ \\
1.0 & 0.540302306 & 0.474175618 & 0.540302306 & $4.76286 \times 10^{-14}$ & $1.97000 \times 10^{-8}$ \\
\hline
\end{tabular}

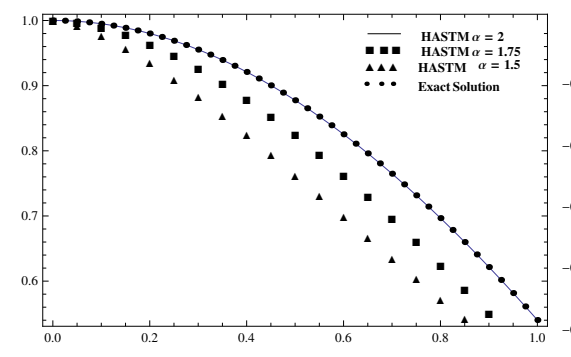

(a)

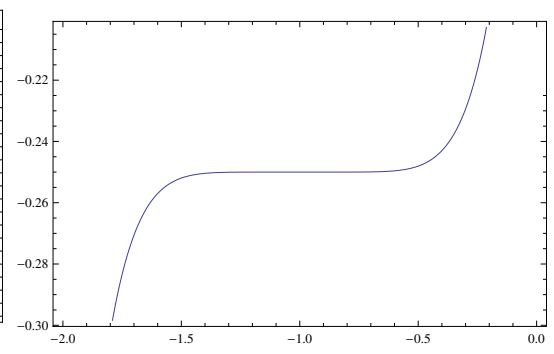

(b)

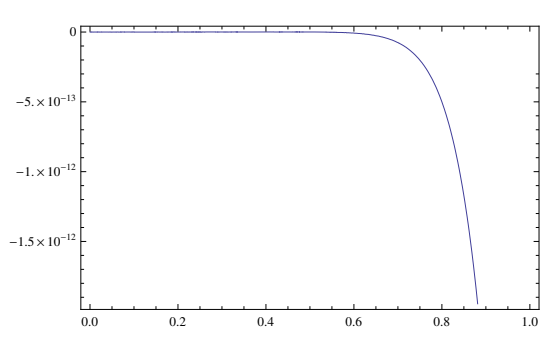

(c)

Figure 3: (a) Comparison between the approximate solution obtained by HASTM at different values of $\alpha$ and the exact one (b)Reprsents the $h$ curve (c) HASTM absolute error

than the approximate solution obtained by modified laguerre wavelets method. Fig. 3 displays the plot of the exact solution and the approximate solutions for some values of $\alpha$, and also represents the plot of the absolute error and the $\mathrm{h}$ - curve.

\section{5. conclusions}

In this research article, HASTM has been employed successfully for the first time to obtain a new accurate approximate solution for a classes fractional delay differential equation. It has been found that the construction of this procedure has a very rapid convergent series solution due to the general formula of its coefficients which can be determined after a few successive iterations. This procedure has been tested upon several examples of linear and nonlinear FDDes and gives a good approximation in few terms which is converged to the exact solution.

\section{References}

[1] T. Al-Hawary, A. A. Amourah \& M. Darus, Differential sandwich theorems for p-valent functions associated with two generalized differential operator and integral operator. International Information Institute (Tokyo). Information, 17(8) (2014), 3559. 1

[2] A. K. Alomari, N. R. Anakira \& I. Hashim,. Multiple solutions of problems in fluid mechanics by predictor optimal homotopy asymptotic method. Advances in Mechanical Engineering, 6(2014), ID372537. https:/ / doi.org/10.1155/2014/372537 1 
[3] A. K. Alomari, N. R. Anakira, A. S. Bataineh \& I. Hashim, Approximate solution of nonlinear system of BVP arising in fluid flow problem. Mathematical Problems in Engineering, 2013. https:// doi.org/10.1155/2013/136043 1

[4] A. A. Amourah \& F. Yousef, Some properties of a class of analytic functions involving a new generalized differential operator. Boletim da Sociedade Paranaense de Matemca, 38(6)(2019), 33-42. https:/ / doi.org/10.5269/bspm.v38i6.40530 1

[5] A. A. Amourah, Initial bounds for analytic and bi-univalent functions by means of $(p, q)$ - Chebyshev polynomials defined by differential operator. General Letters in Mathematics, 7 (2)(2019), 45-51. https:// doi.org/10.31559/glm2019.7.2.1 1

[6] N. R. Anakira, A. Jameel, A. K. Alomari, A. Saaban, M. Almahameed, \& I. Hashim, Approximate solutions of multipantograph type delay differential equations using multistage optimal homotopy asymptotic method. Journal of Mathematical and Fundamental Sciences, 50(3)(2018), 221-232. https://doi.org/10.1155/2013/498902 1

[7] N. R. Anakira, M. M. Al-Shorman \& A. F. Jameel,An Accurate Approximate Solutions of Multipoint Boundary Value Problems.General Letters in Mathematics, 7(1)(2019),31-38. https://doi.org/10.31559/glm2019.7.1.4 1

[8] N. R. Anakira, A. K. Alomari \& I. Hashim,Application of optimal homotopy asymptotic method for solving linear delay differential equations. In AIP Conference Proceedings, 1571 (1)(2013),1013-1019. American Institute of Physics. https:/ / doi.org/10.1063/1.4858786 1

[9] N. R. Anakira, Optimal homotopy asymptotic method for solving multi-pantograph type delay differential equations. advances in differential equations and control processes, 19(3)(2018), 191-204. https:/ / doi.org/10.17654/de019030191 1

[10] N. Anakira, Solution of system of ordinary differential equations by optimal homotopy asymptotic method. In AIP Conference Proceedings (Vol. 2096, No. 1, p. 020023). AIP Publishing LLC,(2019, April). 1

[11] Y. Asai \& P. E. Kloeden, Numerical schemes for ordinary delay differential equations with random noise. Applied Mathematics and Computation, 347(2019), 306-318. https://doi.org/10.1016/j.amc.2018.11.033 1

[12] W. R. A. AL-Hussein \& S. N. Al-Azzawi,Approximate solutions for fractional delay differential equations by using Sumudu transform method. In AIP Conference Proceedings, Vol. 2096, No. 1, p. 020007. (2019, April). 2, 4.1, 1, 2, 4.2

[13] R. L. Bagley\& P. J. Torvik, Fractional calculus in the transient analysis of viscoelastically damped structures, AIAA journal, 23(6)(1985), 918-925. https: / / doi.org/10.2514/3.9007 1

[14] A. Carpinteri \& F. Mainardi,Fractals and fractional calculus in continuum mechanics,Springer,(Vol. 378)(2014). 1

[15] M. Hamarsheh, A. Ismail \& Z. Odibat, Optimal homotopy asymptotic method for solving fractional relaxationoscillation equation. Journal of Interpolation and Approximation in Scientific Computing, 2(2015), 98-111. https:/ / doi.org/10.5899/2015/jiasc-00081 1

[16] N. Herisanu, V. Marinca, T. Dordea, and G. Madescu, A new analytical approach to nonlinear vibration of an electric machine, Proceedings Of Romanian Academy Series A, 9(3)(2008). 1

[17] M. A. Iqbal, U. Saeed \& S. T. Mohyud-Din, Laguerre wavelets method for delay differential equations of fractionalorder. Egyptian journal of basic and applied sciences, 2(1)(2015), 50-54. 4.3, 3

[18] H. Jafari \& S. Seifi,Homotopy analysis method for solving linear and nonlinear fractional diffusion-wave equation. Communications in Nonlinear Science and Numerical Simulation, 14(5)(2009), $2006-2012$. https: / / doi.org/10.1016/j.cnsns.2008.05.008 1

[19] A. F. Jameel, N. R. Anakira, A. K. Alomari, M. Al-Mahameed \& A. Saaban, A new approximate solution of the fuzzy delay differential equations. International Journal of Mathematical Modelling and Numerical Optimisation, 9(3)(2019), 221-240. https://doi.org/10.1504/ijmmno.2019.100476 1

[20] A. F. Jameel, A. Saaban, S. A. Altaie, N. R. Anakira, A. K. Alomari, \& N. Ahmad, Solving first order nonlinear fuzzy differential equations using Optimal Homotopy Asymptotic Method. International Journal of Pure and Applied Mathematics, 118(1)(2018), 49-64. 1

[21] A. F. Jameel, N. R. Anakira, M. M. Rashidi, A. K. Alomari, A. Saaban \& M. A. Shakhatreh .DifferentialTransformation Method For Solving High Order Fuzzy Initial Value Problems. Italian Journal of Pure and Applied Mathematics, 39(2018), 194-208. 1

[22] A. F. Jameel, N. Anakira, A. K. Alomari, I. Hashim \& S. Momani, A New Approximation Method for Solving Fuzzy Heat Equations. Journal of Computational and Theoretical Nanoscience, 13(11)(2016), 7825-7832. https://doi.org/10.1166/jctn.2016.5784 1

[23] M. M. Khader \& A. S. Hendy, The approximate and exact solutions of the fractional-order delay differential equations using Legendre seudospectral method. International Journal of Pure and Applied Mathematics, 74(3)(2012), $287-297$. 1

[24] Y. Komori, A. Eremin \& K. Burrage, S-ROCK methods for stochastic delay differential equations with one fixed delay. Journal of Computational and Applied Mathematics, 353(2019), 345-354. https://doi.org/10.1016/j.cam.2018.12.042 1

[25] V. V. Kulish \& J. L. Lage,Application of fractional calculus to fluid mechanics, J. Fluids Eng., 124(3)(2002), 803-806. https://doi.org/10.1115/1.1478062 1

[26] C. Lederman, J. M. Roquejoffre\& N. Wolanski, Mathematical justification of a nonlinear integro-differential equation for the propagation of spherical flames. Annali di Matematica Pura ed Applicata, 183(2)(2004), 173-239. https: / / doi.org/10.1007/s10231-003-0085-1 1

[27] O. H. Mohammed \& A. I. Khlaif, Adomian decomposition method for solving delay differential equations of fractional 
order. structure, 12(13)(2014), 14-15. 1

[28] N. Ratib Anakira, A. K. Alomari, A. K. \& I. Hashim .Optimal homotopy asymptotic method for solving delay differential equations. Mathematical Problems in Engineering,(2013), 1-11. https://doi.org/10.1155/2013/498902 1

[29] U. Saeed, Hermite wavelet method for fractional delay differential equations. Journal of Difference Equations, 2014 (2014),1-8. https://doi.org/10.1155/2014/359093 1

[30] L. Song \& H. Zhang,Application of homotopy analysis method to fractional KdVBurgersKuramoto equation. Physics Letters A, 367(2007), 88-94. 1

[31] M. Q. Xu \& Y. Z. Lin,Simplified reproducing kernel method for fractional differential equations with delay. Applied Mathematics Letters, 52(2016), 156-161. https://doi.org/10.1016/j.aml.2015.09.004 1 